\title{
Time-to-Event Analysis of Paclitaxel-Associated Peripheral Neuropathy in Advanced Non-Small-Cell Lung Cancer Highlighting Key Influential Treatment/Patient Factors $\mathbf{s}$
}

\author{
Francis W. Ojara, Andrea Henrich, Nicolas Frances, Wilhelm Huisinga, Niklas Hartung, \\ Markus Joerger, and Charlotte Kloft \\ Department of Clinical Pharmacy and Biochemistry, Institute of Pharmacy, Freie Universitaet Berlin, Germany (F.W.O., A.H., \\ C.K.); Graduate Research Training Program PharMetrX, Germany (F.W.O., A.H.); Department of Translational Modeling and \\ Simulation, Roche Pharma Research and Early Development, Roche Innovation Center Basel, F. Hoffmann-La Roche Ltd., Basel, \\ Switzerland (N.F.), Institute of Mathematics, University of Potsdam, Potsdam, Germany (N.H, W.H.); and Department of Oncology \\ and Hematology, Cantonal Hospital, St. Gallen, Switzerland (M.J.)
}

Received April 14, 2020; accepted September 3, 2020

\begin{abstract}
Paclitaxel-associated peripheral neuropathy (PN), a major doselimiting toxicity, significantly impacts patients' quality of life/ treatment outcome. Evaluation of risk factors often ignores time of PN onset, precluding the impact of time-dependent factors, e.g., drug exposure, needed to comprehensively characterize PN. We employed parametric time-to-event (TTE) analysis to describe the time course of risk of first occurrence of clinically relevant $\mathrm{PN}$ grades $\geq 2$ (PN2+, $n=105$, common terminology criteria v4.0) and associated patient/treatment characteristics, leveraging data from 365 patients (1454 cycles) receiving paclitaxel every 3 weeks (plus carboplatin AUC $=6$ or cisplatin $80 \mathrm{mg} / \mathrm{m}^{2}$ ) for $\leq 6$ cycles. Paclitaxel was intravenously administered ( 3 hours) as standard $200-\mathrm{mg} / \mathrm{m}^{2}$ doses $(n=182)$ or as pharmacokinetic-guided dosing $(n=183)$. A cycle-varying hazard TTE model linking surge in hazard of PN2+ to paclitaxel administration [PN2+ proportions (i.e., cases per 1000 patients), $1^{\text {st }}$ day, cycle 1: 4.87 of 1000 ; cycle $6: 7.36$ of 1000 ] and linear decline across cycle (last day, cycle 1: 1.64 of 1000; cycle 6: 2.48 of 1000 ) adequately characterized the time-varying hazard of PN2+. From joint covariate evaluation, PN2+ proportions $\left(1^{\text {st }}\right.$ day, cycle 1) increased by 1.00 per 1000 with $5-\mu \mathrm{mol} \cdot \mathrm{h} / \mathrm{l}$ higher paclitaxel exposure per cycle (AUC between the start and end of a cycle, most relevant covariate), 0.429 per 1000 with 5-year higher age, 1.31 per 1000 (smokers vs. nonsmokers), and
\end{abstract}

decreased by 0.670 per 1000 (females vs. males). Compared to $200 \mathrm{mg} / \mathrm{m}^{2}$ dosing every 3 weeks, model-predicted cumulative risk of PN2+ was significantly higher $(42 \%)$ with $80 \mathrm{mg} / \mathrm{m}^{2}$ weekly dosing but reduced by $11 \%$ with $175 \mathrm{mg} / \mathrm{m}^{2}$ dosing every 3 weeks. The established TTE modeling framework enables quantification and comparison of patient's cumulative risks of PN2+ for different clinically relevant paclitaxel dosing schedules, sparing patients PN2+ to improve paclitaxel therapy.

\section{SIGNIFICANCE STATEMENT}

Characterization of risk factors of paclitaxel-associated peripheral neuropathy (PN) typically involves time-independent comparison of PN odds in patient subpopulations, concealing the impact of time-dependent factors, e.g., changing paclitaxel exposure, required to comprehensively characterize PN. We developed a parametric time-to-event model describing the time course in risk of clinically relevant paclitaxel-associated PN, identifying the highest risk in older male smokers with higher paclitaxel area under the plasma concentration-time curve between the start and end of a cycle. The developed framework enabled quantification of patient's risk of PN for clinically relevant paclitaxel dosing schedules, facilitating future dosing decisions.

\section{Introduction}

Peripheral neuropathy (PN) is a major cumulative, often irreversible, dose-limiting toxicity of paclitaxel with significant

There was no outside funding for this paper. C.K and W.H. report grants from an industry consortium (AbbVie Deutschland $\mathrm{GmbH} \& \mathrm{Co}$. KG, Boehringer Ingelheim Pharma GmbH \& Co. KG, Grünenthal GmbH, F. Hoffmann-La Roche Ltd., Merck KGaA, AstraZenaca UK Limited and Sanofi) for the PharMetrX program. C.K. reports grants for the Innovative Medicines Initiative-Joint Undertaking [Drug Disease Modelling Resources (DDMoRe)], Diurnal Ltd., the Federal Ministry of Education and Research within the Joint Programming Initiative on Antimicrobial Resistance Initiative (JPIAMR) and from the European Commission within the Horizon 2020 framework programme ("FAIR"), all outside the submitted work.

https://doi.org/10.1124/jpet.120.000053.

S This article has supplemental material available at jpet.aspetjournals.org. impact on patients' quality of life and may influence treatment outcome (Stubblefield et al., 2009). Over $20 \%$ of patients receiving standard paclitaxel dosing $\left(175-200 \mathrm{mg} / \mathrm{m}^{2}\right.$, every 3 weeks) against non-small-cell lung cancer (NSCLC) experience clinically relevant PN (Joerger et al., 2016; Zhang et al., 2019). The currently accepted mechanism of paclitaxelassociated PN is through microtubule hyperstabilization, distorting the physiologic cycle of microtubule depolymerization and repolymerization and subsequently interfering with axonal growth, intracellular transport, and the structural integrity of neurons (Mielke et al., 2005; Gornstein and Schwarz, 2017). Paclitaxel-associated PN typically manifests with sensory symptoms, such as pain, paresthesia, dysesthesia, and numbness, primarily in the hands and feet, beginning 
as early as 24-72 hours after administration of paclitaxel (Scripture et al., 2006; Boyette-Davis et al., 2013).

The risk of $\mathrm{PN}$ has been shown to increase with higher paclitaxel doses by comparing proportions of $\mathrm{PN}$ in patients receiving different paclitaxel doses (Green et al., 2005; Seidman et al., 2008) and higher systemic exposure, i.e., time of paclitaxel plasma concentration above $0.05 \mu \mathrm{M}\left(\mathrm{T}_{\mathrm{C}>0.05 \mu \mathrm{M}}\right)$ and area under the concentration-time curve based on Kaplan-Meier analysis and logistic regression analysis (Mielke et al., 2005; Kraff et al., 2015). Duloxetine, a serotonin and norepinephrine reuptake inhibitor, is currently the only recommended treatment option for chemotherapy-induced PN symptoms (Hershman et al., 2014). However, up to $44 \%$ of the patients with paclitaxel-induced $\mathrm{PN}$ treated with duloxetine experience no relief (Otake et al., 2015), further emphasizing the limited treatment options.

Chemotherapy-induced PN is commonly measured using the National Cancer Institute-Common Terminology Criteria for Adverse Events (NCI-CTCAE) (https://ctep.cancer.gov). Symptoms of PN are graded in order of increasing severity from grades 0 (normal) to 4 (severe) or 5 (death). NCICTCAE PN grades $\geq 2$ are considered clinically relevant, as the inflicted patient may require anticancer treatment delay or dose reductions (Park et al., 2017). To identify factors associated with increased risk of PN, the odds of occurrence of $\mathrm{PN}$ have been compared for different patient characteristics, including age- or treatment-related factors, such as paclitaxel exposure (Abraham et al., 2014; Kraff et al., 2015; Tanabe et al., 2017). These statistical analyses, however, ignore the influence of the time of onset of PN in describing the risk of PN and potentially conceal the underlying time-related pathophysiological or pharmacological processes, e.g., change in drug exposure over time, required to accurately characterize the occurrence of PN.

Time-to-event analysis (TTE) (Holford, 2013) provides a framework to integrate the impact of time of occurrence of PN while describing the occurrence of PN. In parametric TTE analysis, the time course in probability of $\mathrm{PN}$ is described using parameters of a TTE model. Prior knowledge of biologic and pharmacological processes associated with the occurrence of $\mathrm{PN}$ can be integrated in the model, hence providing mechanistically plausible and more accurate description of observed PN data. Parametric TTE models can further be used to simulate and predict the risk of events such as PN at specific time points for patients with specific covariate characteristics or dosing regimens (Lu et al., 2017; Svensson et al., 2018).

This work aimed to 1) describe the time course in risk of first occurrence of clinically relevant paclitaxel-associated PN using parametric TTE analysis; 2) evaluate the impact of paclitaxel exposure and relevant patient characteristics on the risk of clinically relevant $\mathrm{PN}$; and 3) generate inference on the risk of clinically relevant $\mathrm{PN}$ for different paclitaxel dosing schedules to manage the risk of PN.

\section{Methods}

Demographic and Clinical Data. We analyzed clinical data from the CEPAC-TDM study, an open-label, randomized, phase III, multicenter study (clinicaltrials.gov identifier NCT01326767) (Joerger et al., 2016). Briefly, 365 patients with newly diagnosed advanced NSCLC received paclitaxel (3-hour intravenous infusion) plus carboplatin (target AUC $=6 \mathrm{mg} \cdot \mathrm{min} / \mathrm{ml}$ ) or cisplatin $\left(80 \mathrm{mg} / \mathrm{m}^{2}\right)$ every 3 weeks for a maximum of six cycles. In the conventional, body surface area (BSA)-guided dosing arm, 182 patients (734 treatment cycles) received the standard paclitaxel dose of $200 \mathrm{mg} / \mathrm{m}^{2}$, whereas in the experimental pharmacokinetic (PK)-guided dosing arm, 183 patients (720 treatment cycles) received PK-guided dosing of paclitaxel according to an algorithm based on paclitaxel exposure $\left(\mathrm{T}_{\mathrm{C}>0.05}\right.$ $\mu \mathrm{M}$ ) from the previous cycle (Joerger et al., 2012). PK sampling was performed only in the PK-guided dosing arm, and $\mathrm{T}_{\mathrm{C}>0.05 \mu \mathrm{M}}$ was determined by post hoc estimation based on a paclitaxel PK model (Joerger et al., 2012). Median patient age was 64 years (range 41-78), and the proportions of females and current smokers were $33 \%$ and $37 \%$, respectively. A detailed summary of the dosing algorithm and patient demographic and clinical characteristics has been published (Joerger et al., 2016). PN symptoms, severity, date of onset, and date of resolution were documented during study visits and graded according to NCI-CTCAE version 4.0 (https://ctep.cancer.gov).

Time-to-Event Model of Paclitaxel-Associated PN. Based on clinical judgment and literature (Park et al., 2017), PN data were dichotomized, with grade 1 categorized as clinically nonrelevant and grades $\geq 2$ (PN2+) as clinically relevant. The first occurrence of PN2+ (incidence) within each patient was considered an event. Parametric time-to-event analysis was employed to describe the risk of PN2+ over time. In this setting, the actual time of first occurrence of PN2+ in an individual, $t$, was regarded as the value of a random variable, T. A hazard-based approach was used in which the distribution of $\mathrm{T}$ was specified through a parametric hazard function, $\mathrm{h}(\mathrm{t})$, obtained from the probability that a patient experienced first occurrence of PN2+ at time $t$. Based on $h(t)$, the probability density function (pdf) of $T$ can be derived, as well as the so-called survivor function $[S(t)]$, which described the probability that an individual had not experienced the event before a given time $t$. Whenever a patient experienced an incidence of $\mathrm{PN} 2+$, the likelihood of the event at that time was calculated using the pdf of $\mathrm{T}$, whereas if a patient never experienced $\mathrm{PN} 2+$, the survivor function corresponding to the final day of the last treatment cycle (censoring time) was calculated. The mathematical formulas linking $\mathrm{h}(\mathrm{t}), \mathrm{S}(\mathrm{t})$, and the pdf of $\mathrm{T}$ at time $\mathrm{t}$ are given as follows:

$$
\begin{gathered}
h(t)=\lim _{\delta t \rightarrow 0}\left\{\frac{P(t \leq T<t+\delta t)}{\delta t}\right\} \\
S(t)=\exp \left(-\int_{0}^{t} h(u) \cdot d u\right) \\
\operatorname{pdf}(t)=S(t) \times h(t),
\end{gathered}
$$

in which $\mathrm{u}$ is a variable for integration by substitution and standard notation for integration.

Different baseline hazard functions (constant, Weibull, and Gompertz) were investigated for their ability to describe the risk of first occurrence of PN2+ over time. All these functions describe a monotonic change in hazard over time. Given the knowledge on PN pathophysiology, i.e., substantial damage of the neurons 1-3 days after exposure to clinically relevant concentrations of paclitaxel (Gornstein and

ABBREVIATIONS: AUC area under the plasma concentration-time curve $\mathrm{AUC}_{\text {cycle, }}$ area under the plasma concentration-time curve between the start and end of a cycle; BSA, body surface area; CEPAC-TDM, Central European Society of Anticancer Drug Research Study of Paclitaxel Therapeutic Drug Monitoring; Cl, confidence interval; CIPN, chemotherapy-induced PN; $F$, hazard surge scale factor; FCM, full covariate model; LRT, likelihood ratio test; NCI-CTCAE, National Cancer Institute-Common Terminology Criteria for Adverse Events; NSCLC, non-small-cell lung

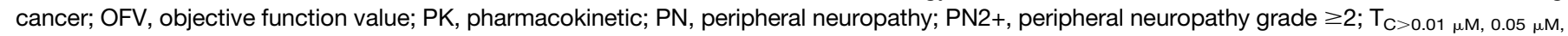
$0.1 \mu \mathrm{M}$, time of paclitaxel plasma concentrations above thresholds of $0.01,0.05$, and $0.1 \mu \mathrm{M}$, respectively; TTE, time-to-event. 
Schwarz, 2017), and observations in the CEPAC-TDM study, i.e., a higher proportion of patients experiencing PN2+ within the first few days after paclitaxel administration, we considered in addition a "cycle-varying hazard model" to allow for a cycle-specific change in hazard over time. In this model, a surge in hazard was linked to the administration of paclitaxel and estimated as a hazard surge term $F$. In this analysis, $F$ was estimated by scaling a unit hazard introduced into the hazard compartment at the time of paclitaxel administration (Supplemental Fig. 1). Across a cycle, from the first to the last day, the hazard declined following a first-order process described by the rate constant $K$. Equation 4 and 5 below show the cycle-varying hazard model:

$$
\begin{gathered}
\mathrm{h}\left(\mathrm{t}_{\text {Dose }}\right)=\mathrm{h}_{\text {Previous }}+\mathrm{F} ; \quad \text { at the cycle start } \\
\frac{\mathrm{dh}(\mathrm{t})}{\mathrm{dt}}=-K \cdot \mathrm{h}(\mathrm{t}) \quad \text { within cycle, }
\end{gathered}
$$

in which $\mathrm{h}\left(\mathrm{t}_{\text {Dose }}\right)$ and $\mathrm{h}_{\text {Previous }}$ (both in day ${ }^{-1}$ ) are the hazards of first occurrence of PN2+ at the beginning of a cycle (cycle day 1 ) and the end of the previous cycle, respectively (for cycle $1, h_{\text {Previous }}=0$ ); $h(t)$ (in day $^{-1}$ ) is the hazard of first occurrence of $\mathrm{PN} 2+$ at any cycle time $t ; F$ is the hazard surge term describing the increase in hazard of first occurrence of $\mathrm{PN} 2+$ at the start of a cycle, and $K$ is the first-order hazard decay rate constant describing the decline in hazard of first occurrence of PN2+ over time within each cycle.

Impact of Covariates on Hazard of First Occurrence of PN2+. Paclitaxel PK data were only available in the PK-guided dosing arm; however, we aimed to evaluate the impact of paclitaxel PK on PN for the entire data set. As such, individual patient paclitaxel exposure metrics, i.e., time of plasma concentrations above thresholds, $0.01,0.05$, and $0.1 \mu \mathrm{M}\left(\mathrm{T}_{\mathrm{C}>0.01 \mu \mathrm{M}}, \mathrm{T}_{\mathrm{C}>0.05 \mu \mathrm{M}}\right.$, and $\mathrm{T}_{\mathrm{C}>0.1 \mu \mathrm{M}}$, respectively), and area under the plasma concentration-time curve between the start and end of a cycle $\left(\mathrm{AUC}_{\text {cycle }}\right)$ were derived by imputation (single and multiple) based on a PK model developed from the PK-guided dosing arm data (Henrich et al., 2017) and individual patient data (Supplemental Data). The imputation strategy was validated by comparing distributions of imputed and estimated exposure in the PK-guided dosing arm, both at individual and population levels.

Covariate analysis was performed in two steps: first, covariates were univariately evaluated with respect to $F$ and $K$ (based on paclitaxel exposure from single imputation) to independently determine the paclitaxel exposure metric most predictive of PN2+. Parameter-covariate relations were evaluated using the linear, proportional, and exponential models. Secondly, paclitaxel exposure, age, sex, and smoking status were jointly evaluated in a full covariate model (FCM) (Gastonguay, 2011) based on their known mechanistic pharmacological link to peripheral neuropathy. Age-related changes in nerve fibers, such as axonal atrophy, and electrophysiological changes, such as impaired nerve regeneration after injury, have been described (Verdú et al., 2000). Estrogen-mediated increase in proinflammatory cytokines and testosterone-mediated production of antiinflammatory cytokines are linked to stronger immune response and higher sensitivity to neuropathic pain in females compared with males (Rosen et al., 2017). Smoking was found to impair functional recovery subsequent to peripheral nerve injury (Rinker et al., 2011). These covariates were also significantly associated with peripheral neuropathy in the clinical setting (Kawakami et al., 2012; de Graan et al., 2013; Kanbayashi et al., 2013). The final FCM parameters were derived after multiple imputation (50 replicates) of paclitaxel exposure (Johansson and Karlsson 2013; Svensson et al., 2018). The dependence of covariate effects of paclitaxel exposure, age, sex, and smoking status on treatment arm (BSA-guided or PK-guided dosing) was assessed by estimating the FCM parameters with and without treatment arm as a covariate. Subsequently, based on the estimated FCM parameters, 250 virtual clinical trials were simulated for a standard paclitaxel dosing schedule, varying the level of a specific covariate while keeping other covariates at reference value, and cumulative proportions of $\mathrm{PN} 2+$ were computed at the end of treatment.

Time-to-Event Model Evaluation. To compare the different baseline hazard models and evaluate the FCM, Kaplan-Meier visual predictive checks were used: 250 data sets were generated by simulation from the developed models, followed by graphical comparison of the simulated and observed data. In univariate covariate analysis, the likelihood ratio test (LRT) was used to assess statistical significance of covariates. For any two nested models, e.g., the baseline TTE model (with no covariates) and a covariate model, a change in objective function value of $\geq 3.84$ points as numeric quality criterion for covariate inclusion showed statistical significance of the covariate effect, corresponding to an asymptotic type 1 error of $\alpha=0.05\left(\chi^{2}\right.$ distribution with one degree of freedom, corresponding to one additional model parameter).

Risk of PN2+ with Different Paclitaxel Dosing Schedules. Exploiting the developed TTE full covariate model, simulations were performed to evaluate the risk of first occurrence of PN2+ in three clinically relevant paclitaxel dosing schedules for NSCLC (summarized in Table 1). A virtual population of 1000 patients with NSCLC was generated by sampling with replacement from the distribution of patient characteristics in the CEPAC-TDM data base: sex and smoking status were sampled empirically (i.e., without consideration for the distribution of other patient characteristics), whereas age was sampled based on the distribution within the respective sex. All three dosing schedules were administered to each patient, one at a time, and typical paclitaxel PK exposure was derived using the paclitaxel PK model (Henrich et al., 2017). Parameters (250 sets) of the FCM without treatment arm effect were sampled from their uncertainty distributions, and for each set, a clinical trial was simulated based on the virtual populations. Hence, model parameter uncertainty and randomness of the time-to-event model were the two levels of variability included during the simulations. As outcome, 1) the simulated proportion of patients who experienced $\mathrm{PN} 2+$ was compared between the different dosing schedules across time during treatment, and 2) risk ratios between the different dosing schedules were computed based on simulated proportions of PN2+ at the end of treatment of each trial.

Software. Data set preparation and statistical evaluation were performed in R 3.4.3 (https://www.r-project.org), and TTE analysis was performed with the first-order method in NONMEM 7.3.0 with assistance of PsN 4.2.0 (Lindbom et al., 2005) and Pirana 2.9.4 (Keizer et al., 2011).

\section{Results}

Exploratory PN Data Analysis. In total, 105 patients (28.8\%) experienced a first occurrence of PN2+ during any treatment cycle. Generally, a higher number of events were observed in the first few days subsequent to paclitaxel treatment administration (cycle start), with the numbers gradually declining across the cycle in all observed cycles (Fig. 1, solid line). The number of events recorded on day 1 of a cycle increased across cycles; no event was recorded on day 1 of the first cycle. No specific trend in proportion of incidence of PN2+ across treatment cycles was apparent; however, cycle 1 had the lowest proportion of PN2+ (5.6\%), with cycle 4 having the highest proportion of $\mathrm{PN} 2+(14.6 \%)$.

TABLE 1

Selected clinically relevant paclitaxel dosing schedules

\begin{tabular}{lc}
\hline Schedule & Dosing Frequency \\
\hline $200 \mathrm{mg} / \mathrm{m}^{2}$ every 3 weeks & Day 1 of a 21-day cycle, 6 cycles \\
$175 \mathrm{mg} / \mathrm{m}^{2}$ every 3 weeks & Day 1 of a 21-day cycle, 6 cycles \\
$80 \mathrm{mg} / \mathrm{m}^{2}$ once every week & Days $1,8,15$ of a 28-day cycle, 6 cycles \\
\hline
\end{tabular}




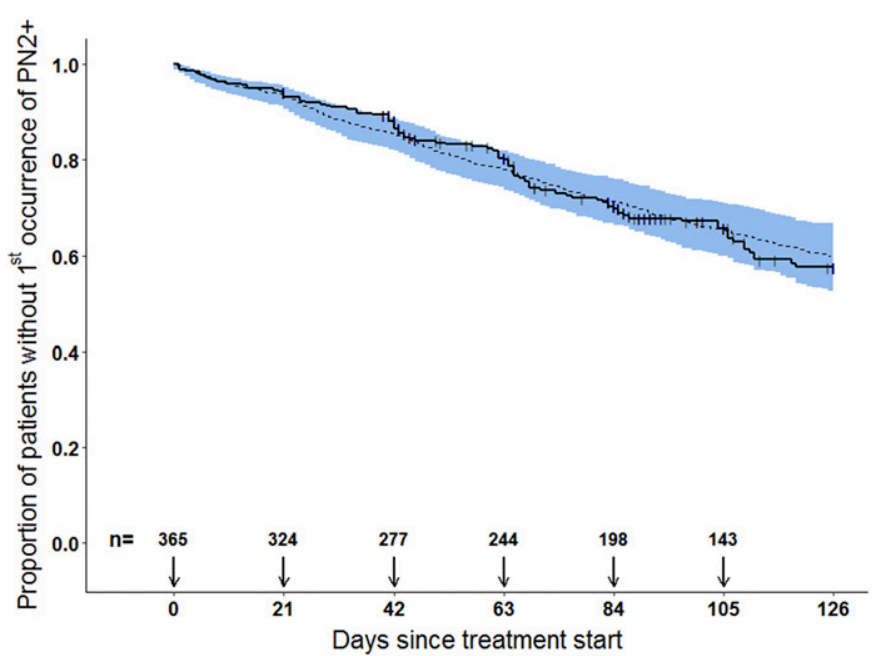

Fig. 1. Kaplan-Meier visual predictive check for the cycle-varying hazard baseline time-to-event model showing observed and model prediction of first occurrence of PN2+. Proportion of patients without first occurrence of PN2+ is plotted across time, with observations censored at the last protocol treatment time. Solid line: observed data (vertical lines: censor times due to dropout from various reasons); dashed line: median modelpredicted profile, $90 \%$ CI (blue shade); $n$ is the number of patients at specific observation times; arrows: day of planned paclitaxel administration as specified in the study protocol.

Of the 260 patients who did not experience PN2+, 75 received six cycles of treatment (meaning they did not drop out but were censored at the end of the study period), whereas the remaining 185 patients were considered dropouts (censored at dropout) with regard to $\mathrm{PN} 2+$ analysis. The reasons for dropout included tumor progression (43.0\%), patient death (13.0\%), and among others, cessation of treatment at physician's discretion, withdrawal of consent, and intolerable non-neurologic toxicities. Therefore, dropouts were assumed random with regard to $\mathrm{PN} 2+$ and not explicitly accounted for in developing this time-to-event model of peripheral neuropathy.

Time-to-Event Model of Paclitaxel-Associated PN2+. Based on the LRT, no statistically significant difference in model fit was found between the constant, Weibull, and Gompertz hazard models (change in OFV $<3.84$ points between the three models but 24.4 higher than cycle-varying hazard model, Supplemental Table 1). However, as the cycle-varying hazard and constant hazard models were not nested, these models were compared using visual predictive checks (Supplemental Fig. 2, A-D), rather than the LRT. The cycle-varying hazard model best characterized the observed profile of first occurrence of $\mathrm{PN} 2+$ across time. In contrast to the constant, Weibull, and Gompertz hazard models (Supplemental Fig. 3, A-C), the cycle-varying hazard model (Supplemental Fig. 3D) described an increase in hazard of PN2+ at cycle start, followed by the gradual decrease within the cycle. As such, the cycle-varying hazard model was adopted for subsequent analyses.

Impact of Covariates on the Hazard of first Occurrence of PN2+. Paclitaxel exposure from single imputation closely matched the exposure estimated based on paclitaxel PK, and both fell largely within the interquartile range of paclitaxel exposure from multiple imputation by comparison at both individual and population levels (Supplemental Figs. 4 and 5), demonstrating reliability of the imputation procedure. Hence, individual imputed paclitaxel exposure was adopted in both the BSA-guided and PK-guided dosing arms for further covariate evaluation. Generally, paclitaxel exposure (paclitaxel $\mathrm{AUC}_{\text {cycle }}, \mathrm{T}_{\mathrm{C}>0.01 \mu \mathrm{M}}, \mathrm{T}_{\mathrm{C}>0.05 \mu \mathrm{M}}$, and $\mathrm{T}_{\mathrm{C}>0.1 \mu \mathrm{M}}$ ) was not constant, mainly as a result of dose adaptations in the PKguided dosing arm, but gradually declined across treatment cycles with increase in variability between patients $\left(\mathrm{AUC}_{\text {cycle }}\right.$ and $\mathrm{T}_{\mathrm{C}>0.05 \mu \mathrm{M}}$ shown in Fig. 2).

Paclitaxel AUC cycle $, \mathrm{T}_{\mathrm{C}>0.01 \mu \mathrm{M}}, \mathrm{T}_{\mathrm{C}>0.05 \mu \mathrm{M}}$, and $\mathrm{T}_{\mathrm{C}>0.1 \mu \mathrm{M}}$ all had a statistically significant impact on the hazard of first occurrence of PN2+ (Table 2) from univariate analysis, whereas trends of increase in hazard of PN2+ were observed with older patients, males compared with females, and current smokers compared with current nonsmokers. Higher paclitaxel exposure was associated with a higher surge in hazard or a lower rate of decline in hazard across time when evaluated against $F$ or $K$, respectively, resulting in a higher risk of PN2+ within a cycle. Based on the LRT, paclitaxel $\mathrm{AUC}_{\text {cycle }}$ on $F$ was associated with the greatest improvement in model fit compared to the base model with no covariates (16.1-point drop in OFV, Table 2). The impact of paclitaxel $\mathrm{AUC}_{\text {cycle }}$ on PN2+ was less significant when evaluated with respect to $K$ (reduction in OFV of 12.1). Joint evaluation of the impact of

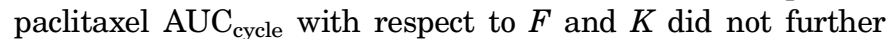
improve model fit compared with $F$ alone, i.e., change in $\mathrm{OFV}$ of 17.3, although it reduced the precision of parameter estimates. This could be attributed to the strong correlation between $F$ and $K$. Furthermore, the condition number for the model with the impact of paclitaxel $\mathrm{AUC}_{\text {cycle }}$ on $\mathrm{F}$ was 84.3 excluding ill conditioning. As such, we retained the impact of paclitaxel $\mathrm{AUC}_{\text {cycle }}$ only on $F$ for subsequent full covariate modeling.

Joint Evaluation of the Impact of Covariates Using a Full Covariate Model. Based on mechanistic pharmacologic plausibility of covariates on $\mathrm{PN}$ and prior clinical knowledge (Kawakami et al., 2012; de Graan et al., 2013; Kanbayashi et al., 2013), age, sex, and smoking status were included for joint evaluation in an FCM. The relationship between covariates and the hazard surge term $(F)$ in the FCM was parameterized as shown in eqs. 6 and 7 for the models without and with treatment arm as covariate, respectively:

$$
\begin{aligned}
& \mathrm{F}=\mathrm{TVF} \cdot \exp \left(E_{\text {AUC }} \cdot\left(\mathrm{AUC}_{\text {cycle }}-\mathrm{AUC}_{(\text {cycle,med })}\right)\right. \\
& \times \cdot \exp \left(E_{\text {Age }} \cdot(\text { Age-Age } \text { med })\right) \cdot\left(E_{\text {Sex }}\right)^{\text {Sex }} \cdot\left(E_{\text {Smok }}\right)^{\text {Smoker }} \\
& \mathrm{F}=\mathrm{TVF} \cdot \exp \left(E_{\text {ARM }} \cdot \mathrm{ARM}\right) \cdot \exp \left(E_{\text {AUC }} \cdot\left(\mathrm{AUC}_{\text {cycle }}-\mathrm{AUC}_{(\text {cycle,med })}\right)\right) \\
& \exp \left(E_{\text {Age }} \cdot\left(\text { Age-Age }_{\text {med }}\right)\right) \cdot\left(E_{\text {Sex }}\right)^{\text {Sex }} \cdot\left(E_{\text {Smok }}\right)^{\text {Smoker }} \text {, }
\end{aligned}
$$

in which TVF is the typical value of $F$ for a male ( $\operatorname{sex}=0$, sex $=1$ for females $)$ current nonsmoker $($ smoker $=0$, smoker $=$ 1 for current smokers $)$ of median age $\left(\right.$ Age $_{\text {med }}=64$ years $)$ and median paclitaxel $\mathrm{AUC}_{\text {cycle }}\left[\mathrm{AUC}_{(\text {cycle, med) }}\right]$; ARM (BSAguided dosing $=0$, PK-guided dosing $=1$ ) is covariate for treatment arm; $E_{\text {AUC }}, E_{\text {Age }}, E_{\text {Sex }}, E_{\text {Smok }}$, and $E_{\text {Arm }}$ are covariate effect coefficients for paclitaxel $\mathrm{AUC}_{\text {cycle, age, sex, }}$ smoking status, and treatment arm, respectively.

Based on parameter estimates of the FCM with no treatment arm effect (eq. 6), an $F$ of 0.00487 day $^{-1}$ (Table 3) was estimated as the hazard on the first day of treatment of a typical patient population [males, current nonsmokers with median age (64 years), and paclitaxel $\mathrm{AUC}_{\text {cycle }}$, which translated into on average 4.87 in 1000 patients experiencing $\mathrm{PN} 2+$. This hazard declined with a first-order rate constant 


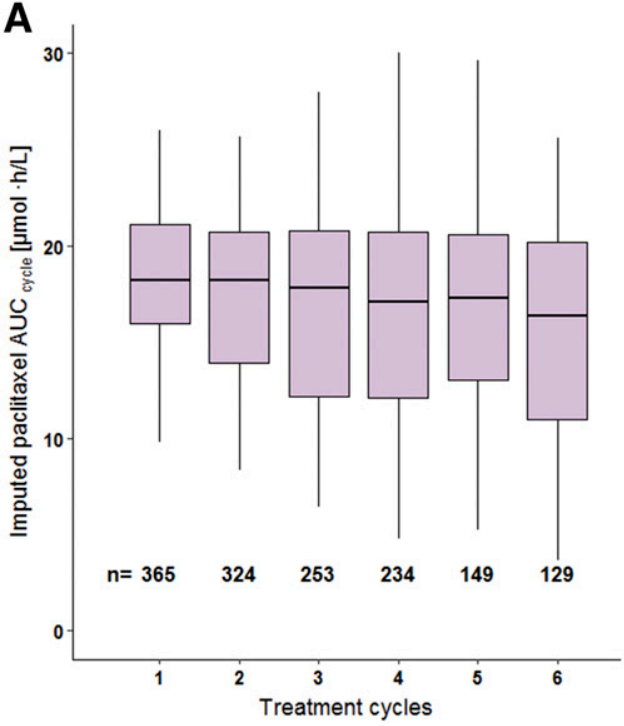

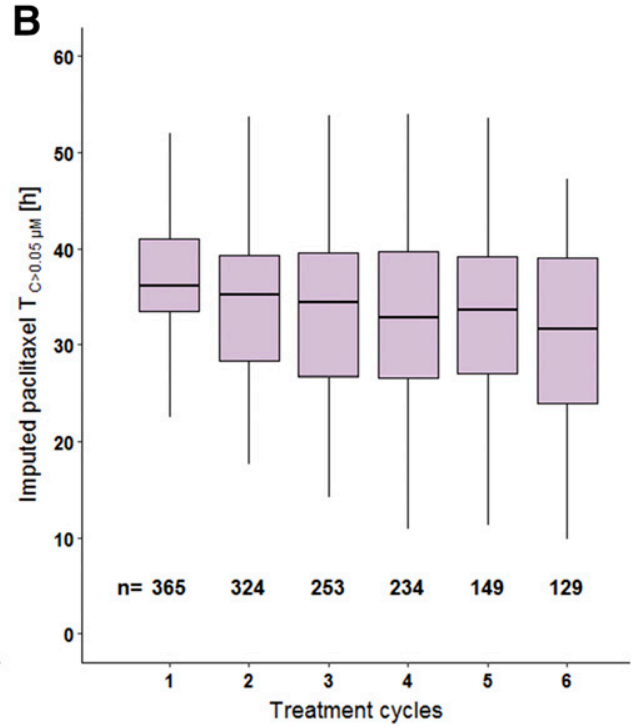

Fig. 2. Distribution of imputed paclitaxel exposure across treatment cycles for the BSA-guided and PK-guided dosing arms combined. Panel A: $\mathrm{AUC}_{\text {cycle; }}$ panel B: time of plasma concentrations above the threshold of $0.05 \mu \mathrm{M}\left(\mathrm{T}_{\mathrm{C}>0.05 \mu \mathrm{M}}\right)$. Boxes: interquartile range (IQR), including median; whiskers (vertical lines): range from box hinge, values within $\pm 1.5 \bullet \mathrm{IQR} ; n$ is the number of patients receiving paclitaxel for a given cycle.
$\left(K=0.0518 \mathrm{day}^{-1}\right)$ to $0.00164 \mathrm{day}^{-1}$ on day 21 (last day of a cycle 1), i.e., 1.64 in 1000 patients experiencing PN2+. For cycle 6 , the hazards on days 1 and 21 were 0.00733 and 0.00247 , respectively, translating into 7.33 in 1000 and 2.47 in 1000 patients experiencing PN2+ on days 1 and 21, respectively. The estimated FCM parameters adequately predicted the observed time-course of of first occurrence of PN2+ (Supplemental Fig. 8).

Covariate impact assessment (by varying a specific covariate while keeping the rest at typical values) revealed a 20\% increase in $F$ (1.00 per 1000 increase in proportions of PN2+) and 43\% increase in $F$ (2.10 per 1000 increase in proportions of $\mathrm{PN} 2+$ ) for a 5 and $10 \mu \mathrm{mol} \cdot \mathrm{h} / \mathrm{l}$ increase in paclitaxel $\mathrm{AUC}_{\text {cycle }}$, respectively; a $9 \%$ increase in $F(0.429$ per 1000 increase in proportions of PN2+) and $18.5 \%$ increase in $F$ (0.900 per 1000 increase in proportions of PN2+) for a 5- and 10 -year increase in age, respectively; and a 27\% higher $F$ (1.31 per 1000 increase in proportions of PN2+) in smokers compared with nonsmokers and a $14 \%$ lower $F(0.670$ per 1000 decrease in proportions of $\mathrm{PN} 2+$ ) in females compared with males. Of note, for the continuous covariates $\mathrm{AUC}_{\text {cycle }}$ and age due to the exponential function of the relation on $F$, a higher exposure and higher age led to a more pronounced effect on $F$, i.e., increase in hazard of first occurrence of $\mathrm{PN} 2+$ at the start of a cycle. Visualization of covariate impact in terms of proportions of patients experiencing PN2+ across time on treatment is shown in Supplemental Fig. 6. Based on the 250 virtual clinical trial simulations, the estimated percentage increase in risk of $\mathrm{PN} 2+$, comparing the $97.5^{\text {th }}$ and $2.5^{\text {th }}$ percentiles of covariate distributions, was $22.0 \%$ and $62.0 \%$ for paclitaxel $\mathrm{AUC}_{\text {cycle }}$ with and without treatment arm effect, respectively, in contrast to $39.0 \%$ and $44.0 \%$ for age with and without treatment arm effect, respectively (Fig. 3), revealing a much stronger dependence of the covariate effect of paclitaxel $\mathrm{AUC}_{\text {cycle }}$ on treatment arm compared with that of age on treatment arm.

Risk of PN2+ with Different Paclitaxel Dosing Schedules. The percentage cumulative risk of $\mathrm{PN} 2+$ at the end of treatment with $200 \mathrm{mg} / \mathrm{m}^{2}$ dosing every 3 weeks was $44.2 \%(90 \% \mathrm{CI}: 32.4 \%-54.8 \%)$ and $64.1 \%(90 \% \mathrm{CI}$ : $45.0 \%-78.3 \%$ ) for the $80 \mathrm{mg} / \mathrm{m}^{2}$ weekly dosing schedule, i.e., the $80 \mathrm{mg} / \mathrm{m}^{2}$ weekly dosing schedule was associated with significantly higher cumulative risk of PN2+ compared with dosing $200 \mathrm{mg} / \mathrm{m}^{2}$ every 3 weeks with a risk ratio of $42.0 \%$ (90\% CI: 9.00\%-90.0\%) (Table 4) after accounting for randomness of the time-to-event model and parameter uncertainty. Although dosing $200 \mathrm{mg} / \mathrm{m}^{2}$ every 3 weeks was associated with a more profound increase in risk of $\mathrm{PN} 2+$ with each paclitaxel administration compared to $80 \mathrm{mg} / \mathrm{m}^{2}$ weekly dosing, the higher dosing frequency in weekly dosing led to a gradual increase in risk of $\mathrm{PN} 2+$ with higher minimum risk of PN2+ after each paclitaxel administration, hence a higher overall cumulative risk of PN2+ (Supplemental Fig. 78). However, a large overlap in proportions of patients experiencing PN2+ was predicted across time between the two dosing schedules (Fig. 4A). For the 175 compared with $200 \mathrm{mg} / \mathrm{m}^{2}$

TABLE 2

Parameter estimates (relative S.E., \%) of the cycle-varying hazard base model and covariate models implementing different imputed paclitaxel pharmacokinetics exposure metrics

\begin{tabular}{|c|c|c|c|c|c|}
\hline Parameter & $\begin{array}{c}\text { Base Model: No } \\
\text { Covariate }\end{array}$ & $\begin{array}{l}\text { Covariate Model with } \\
\mathrm{T}_{\mathrm{C}>0.01 \mu \mathrm{M}}\end{array}$ & $\begin{array}{l}\text { Covariate Model with } \\
\mathrm{T}_{\mathrm{C}>0.05 \mu \mathrm{M}}\end{array}$ & $\begin{array}{l}\text { Covariate Model with } \\
\mathrm{T}_{\mathrm{C}>0.1 \mu \mathrm{M}}\end{array}$ & $\begin{array}{c}\text { Covariate Model with } \\
\text { AUC }_{\text {cycle }}\end{array}$ \\
\hline OFV & 1344.323 & 1337.249 & 1334.563 & 1332.735 & 1328.224 \\
\hline$F$, day $^{-1}$ & $0.00568(35)$ & $0.00500(40)$ & $0.00480(41)$ & $0.00460(41)$ & $0.00460(42)$ \\
\hline$K$, day $^{-1}$ & $0.0568(42)$ & $0.0504(49)$ & $0.0480(52)$ & $0.0477(51)$ & $0.0448(55)$ \\
\hline$E_{C O V}$, covariate unit $^{-1}$ & n.a & $0.0329(43)$ & $0.0456(38)$ & $0.0652(33)$ & $0.0989(29)$ \\
\hline
\end{tabular}

$E_{C O V}$, covariate effect parameter describing change in $F$ with a unit change in paclitaxel exposure relative to the median exposure; $K$, first-order hazard decay rate constant. n.a: not applicable 
TABLE 3

Pooled parameter estimates [(relative S.E., \%), (95\% confidence intervals)] of the cycle-varying hazard full covariate model after multiple imputation

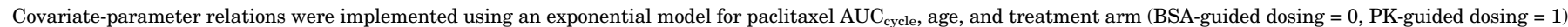

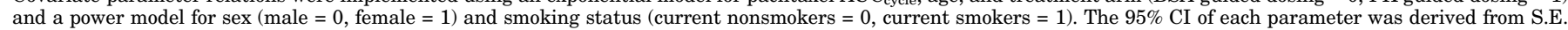
values of estimated parameters by transformation of the variance-covariance matrices. n.a: not applicable

\begin{tabular}{|c|c|c|c|}
\hline Parameter & Parameter Description & Model with No Arm Effect & Model with Arm Effect \\
\hline$F$, day $^{-1}$ & Hazard surge scale factor & $0.00487(42.6),(0.000852,0.00880)$ & $0.00659(43.5),(0.000974,0.0122)$ \\
\hline$K$, day $^{-1}$ & Hazard decay rate constant & $0.0518(47.9),(0.00311,0.100)$ & $0.0531(47.2),(0.00486,0.103)$ \\
\hline$E_{\text {Arm }}$ & Treatment arm effect on $F$ & n.a & $-0.621(41.6),(-1.13,-0.114)$ \\
\hline$E_{A U C}, 1 /(\mu \mathrm{mol} \cdot \mathrm{h} / \mathrm{l})$ & Paclitaxel AUC cycle $_{\text {effect on } F}$ & 0.0359 (51.1), (0.0000320, 0.0718) & $0.00154(151.5),(-0.0308,0.0611)$ \\
\hline$E_{\text {Age }}, \mathrm{y}^{-1}$ & Age effect on $F$ & $0.0169(84.1),(-0.0107,0.0445)$ & $0.0154(91.4),(-0.0122,0.0429)$ \\
\hline$E_{\text {Sex }}$ & Sex effect on $F$ & $0.864(22.2),(0.488,1.23)$ & $0.841(22.0),(0.478,1.20)$ \\
\hline$E_{\text {Smok }}$ & Smoking status effect on $F$ & $1.27(20.4),(0.761,1.78)$ & $1.33(20.2),(0.804,1.85)$ \\
\hline
\end{tabular}

dosing every 3 weeks, an $11.0 \%$ lower risk of PN2+ (90\% CI: $20.5 \%$ decrease, $1.00 \%$ increase) was predicted (Fig. 4B; Table 4), though not statistically significant.

\section{Discussion}

A parametric cycle-varying hazard TTE model based on knowledge of pathophysiology and trends of occurrence of clinically relevant peripheral neuropathy (PN2+) was developed to characterize the time course of the risk of first occurrence of $\mathrm{PN} 2+$ in paclitaxel-treated patients with advanced NSCLC: the risk of PN2+ significantly increased with higher paclitaxel $\mathrm{AUC}_{\text {cycle, }}$, and the most vulnerable population was identified as the older, male, and current smokers from joint evaluation of covariate impacts. The established model enables improved prediction of an individual patient's risk of PN2+ with different clinically relevant paclitaxel dosing schedules for dose selection during treatment.

TTE analysis offers marked improvements over common approaches used for analyzing PN data: traditionally, odds of PN are compared across different patient subpopulations to

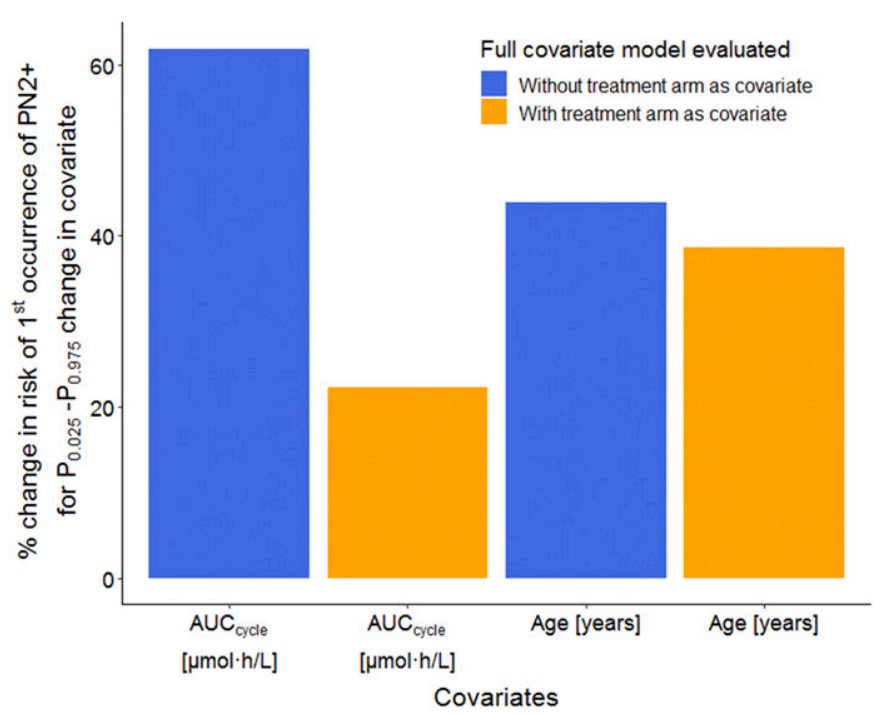

Fig. 3. Increase in risk of first occurrence of PN2+ with change in covariate level. Proportions of first occurrence of PN2+ were simulated at $2.5^{\text {th }}$ percentile $\left(\mathrm{P}_{0.025}\right)$ and $97.5^{\text {th }}$ percentile $\left(\mathrm{P}_{0.975}\right)$ covariate levels using the cycle-varying hazard full covariate models with (orange) and without (blue) treatment arm as a covariate and percentage change in risk of PN2+ calculated. In each case, the covariate of interest was set to the specified percentile while maintaining the remaining covariates at their reference values. ascertain associated risk factors (Scripture et al., 2006; Kanbayashi et al., 2013; Tanabe et al., 2017). These approaches only account for the occurrence of the event without consideration for the time of occurrence and as such conceal knowledge on time-dependent pathophysiological or pharmacological processes required to adequately characterize and predict the occurrence of PN. In the CEPAC-TDM study, a Kaplan-Meier-based nonparametric TTE analysis was used to compare the risk of PN between BSA-guided and PK-guided dosing strategies (Joerger et al., 2016). As an extension, parametric TTE analysis was adopted in this work, which allowed 1) basing on prior knowledge on the pathophysiology of $\mathrm{PN}$ to better describe the baseline hazard of first occurrence of $\mathrm{PN} 2+; 2$ ) a mechanistic evaluation of the impact of nonstratified time-varying covariates, e.g., paclitaxel AUCcycle; and 3) simulation of the occurrence of PN for different paclitaxel exposure levels (dosing regimens) and patient characteristics based on the developed parametric hazard function (TTE model).

The cycle-varying hazard model best described the observed PN2+ data: a surge in incidence of PN2+ at cycle start was linked to the administration of paclitaxel, with the magnitude estimated using the hazard surge term $(F)$. The gradual decline in hazard across time within a cycle was best described as a first-order process, using the hazard decline rate constant $(K)$. Variability in parameters $F$ and $K$ between different patients was linked to differences in patient-specific covariate characteristics. This model structure is supported by in vitro data showing that adult dorsal root ganglion neurons, the cell type inflicted by paclitaxel-associated neurotoxicity, undergo substantial damage 1-3 days after exposure to clinically relevant concentrations of paclitaxel (Gornstein and Schwarz, 2017). This was also consistent with observations from the CEPAC-TDM study, i.e., a higher proportion of patients experienced PN2+ within the first few days after paclitaxel administration.

The effect of paclitaxel exposure could be estimated separately on $F$ and $K$; however, this was not possible on both $F$ and $K$ simultaneously in the same model. Paclitaxel exposure had a stronger effect on $F$ compared with $K$, with $\mathrm{AUC}_{\text {cycle }}$ showing a stronger effect than $\mathrm{T}_{\mathrm{C}>0.01 \mu \mathrm{M}}, \mathrm{T}_{\mathrm{C}>0.05 \mu \mathrm{M}}$, and $\mathrm{T}_{\mathrm{C}>0.1 \mu \mathrm{M}}$. Previous studies also showed a statistically significant increase in the risk of $\mathrm{PN}$ with higher AUC and $\mathrm{T}_{\mathrm{C}>0.05 \mu \mathrm{M}}$ (Mielke et al., 2005; de Graan et al., 2013; Zhang et al., 2016). Unlike these studies, we accounted for cycle-specific changes in exposure, allowing the hazard in an individual to change across cycles, resulting in a more accurate description of the risk of PN2+ across time with changing paclitaxel exposure. The increase in risk of $\mathrm{PN} 2+$ with higher paclitaxel exposure 
TABLE 4

Comparison of risk of first occurrence of PN2+ (90\% confidence intervals) between different clinically relevant paclitaxel dosing schedules

\begin{tabular}{|c|c|c|c|}
\hline \multirow{2}{*}{ Schedule } & \multirow{2}{*}{ Percentage of PN2+ } & \multicolumn{2}{|c|}{ Risk Ratio } \\
\hline & & $175 \mathrm{mg} / \mathrm{m}^{2}$ Every 3 Weeks: $200 \mathrm{mg} / \mathrm{m}^{2}$ Every 3 Weeks & $80 \mathrm{mg} / \mathrm{m}^{2}$ Once Every Week: $200 \mathrm{mg} / \mathrm{m}^{2}$ Every 3 Weeks \\
\hline $200 \mathrm{mg} / \mathrm{m}^{2}$ every 3 weeks & $44.2(32.4,54.8)$ & n.a & n.a \\
\hline $175 \mathrm{mg} / \mathrm{m}^{2}$ every 3 weeks & $39.6(29.4,49.3)$ & $0.891(0.795,1.01)$ & n.a \\
\hline $80 \mathrm{mg} / \mathrm{m}^{2}$ once every week & $64.1(45.0,78.3)$ & n.a & $1.42(1.09,1.90)$ \\
\hline
\end{tabular}

n.a: not applicable

suggests the need for prophylactic management or closer monitoring of symptoms of PN2+ early on within cycles, especially in subpopulations at higher risk of PN2+ (elderly, male, current smokers), to enable early treatment, e.g., with duloxetine (Otake et al., 2015).

Inclusion of treatment arm effect (BSA-guided vs. PKguided) as a covariate on $F$ was associated with a $65 \%$ reduction in the covariate effect of paclitaxel $\mathrm{AUC}_{\text {cycle. This trend was }}$ expected, since protocol dose adaptations in the CEPAC-TDM study led to lower paclitaxel $\mathrm{AUC}_{\text {cycle }}$ in the PK-guided compared to the BSA-guided dosing arm; hence, the observed relationship between treatment arm and $\mathrm{PN} 2+$ is attributed to the difference in paclitaxel $\mathrm{AUC}_{\text {cycle }}$ between the two dosing arms. Treatment arm only partly reduced the covariate effect of paclitaxel $\mathrm{AUC}_{\text {cycle, meaning that paclitaxel } \mathrm{AUC}_{\text {cycle }} \text { was }}$ a stronger predictor of PN2+ compared with treatment arm.

Our findings suggest a higher cumulative risk of PN2+ with $80 \mathrm{mg} / \mathrm{m}^{2}$ weekly dosing compared to $200 \mathrm{mg} / \mathrm{m}^{2}$ paclitaxel dosing every 3 weeks. Weekly dosing was associated with a lower surge in risk of $\mathrm{PN} 2+$ for a single paclitaxel administration; however, a higher cumulative risk of $\mathrm{PN} 2+$ was predicted because of the higher dosing frequency. Previous comparisons of neuropathy risk between weekly dosing and paclitaxel dosing every 3 weeks yielded mixed findings (Green et al., 2005; Schuette et al., 2006; Belani et al., 2008; Seidman et al., 2008; Sparano et al., 2008; Fountzilas et al., 2009). Lower paclitaxel $\mathrm{T}_{\mathrm{C}>0.01 \mu \mathrm{M}}$ with weekly dosing compared with dosing every 3 weeks was associated with reduced toxicity, especially hematologic toxicities (Marchetti et al., 2002). For dosing every 3 weeks, the cumulative risk of PN2+ increased with higher paclitaxel doses, i.e., higher in $200 \mathrm{mg} / \mathrm{m}^{2}$ compared to $175 \mathrm{mg} / \mathrm{m}^{2}$. Ultimately, our results suggest a reduction in risk of $\mathrm{PN} 2+$ with paclitaxel dose reduction rather than dose fractionation (same overall paclitaxel dose administered multiple times within a cycle). Given similar efficacy (progression-free and overall survival) in the dose range of 175-200 mg/m ${ }^{2}$ (Joerger et al., 2016), the lower risk of PN2+ with $175 \mathrm{mg} / \mathrm{m}^{2}$ paclitaxel dosing every 3 weeks suggests an improved therapeutic benefit compared with $200 \mathrm{mg} / \mathrm{m}^{2}$ paclitaxel dosing every 3 weeks.

Alternative time-to-event models that describe a symmetric phase of increase and decrease in hazard of events at predefined times have been employed before for characterizing different events (Plan et al., 2011; Tarning et al., 2014). However, this model structure inadequately described observed PN2+ profiles in this study. Proportions of observed PN2+ peaked a few days after paclitaxel administration and gradually declined over time across the cycle, a profile not consistent with the symmetric model. Alternatively, the hazard of first occurrence of PN2+ could be described using an indirect response model (Upton and Mould, 2014). The paclitaxel concentration-time profiles may be used to drive either the rate of development of the hazard or inhibit the rate of decline of the hazard. The differences in time scale of measurement of paclitaxel concentration and PN2+ events (i.e., hours vs. days) would require fixing PN2+ incidence to a specific clock time, hence introducing a bias in the concentration-PN2+ relationship; as such, we opted for the cycle-varying hazard model and evaluated the time course of incidence of $\mathrm{PN} 2+$ on a time scale of days.

In previous characterization of the time course of chemotherapyinduced PN (CIPN) during paclitaxel treatment, Mehrotra et al. (2017) used a kinetic-pharmacodynamic indirect response model. CIPN scores (0-16) were derived from PN grades from the Functional Assessment of Cancer Therapy/Gynecologic Oncology Group-Neurotoxicity grading system and were
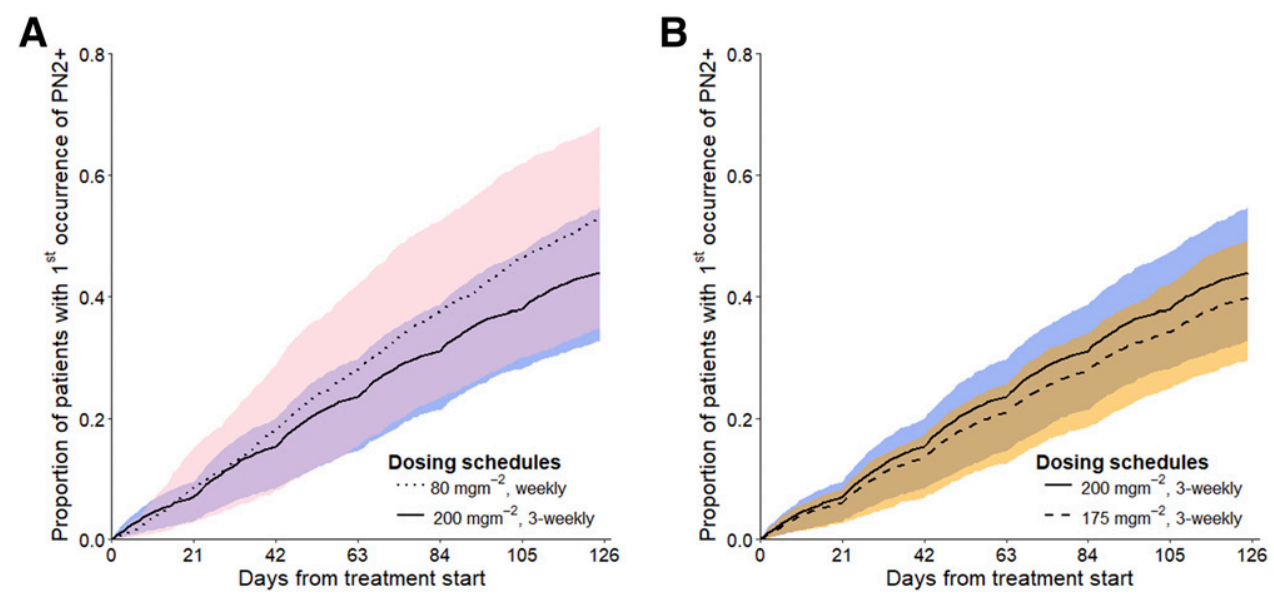

Fig. 4. Cumulative risk of first occurrence of PN2+ across time on treatment. (Panel A) Weekly dosing of $80 \mathrm{mg} / \mathrm{m}^{2}$ vs. $200 \mathrm{mg} / \mathrm{m}^{2}$ dosing every 3 weeks; (panel B) $200 \mathrm{mg} / \mathrm{m}^{2}$ dosing every 3 weeks vs. $175 \mathrm{mg} / \mathrm{m}^{2}$ dosing every 3 weeks. Shades represent $90 \%$ CI of simulated proportion of patients experiencing first occurrence of PN2+ across time on treatment. Pink: $80 \mathrm{mg} / \mathrm{m}^{2}$ weekly dosing; blue: $200 \mathrm{mg} / \mathrm{m}^{2}$ dosing every 3 weeks; orange: $175 \mathrm{mg} / \mathrm{m}^{2}$ dosing every 3 weeks. 
treated as a continuous variable; a score of 4.00 could represent mild experience of each of the four items (score =1). Our data base contained NCI-CTCAE PN grades 1-3 (only three categories) and hence cannot be modeled as a continuous variable. Our model-predicted proportion of PN2+ after six cycles of weekly $80 \mathrm{mg} / \mathrm{m}^{2}$ paclitaxel dosing was $64.1 \%$ (90\% CI: $45.0 \%-78.3 \%$ ), whereas the kinetic-pharmacodynamic model-predicted CIPN scores $\geq 4.00$ was $45.6 \%$ for a patient with BSA of $1.87 \mathrm{~m}^{2}$.

As a possible limitation, our model did not account for the impact of the potentially neurotoxic coadministered platinum drugs. Cisplatin is more neurotoxic than carboplatin, with neuropathy incidences of $28 \%-100 \%$ compared with $6 \%-42 \%$ with carboplatin (Stubblefield et al., 2009). Our data base contained only PK for paclitaxel. In univariate covariate evaluation with respect to $F$ or $K$, platinum drug type had no statistically significant impact, with covariate impacts estimated with unreliably wide confidence intervals. The most probable reason was the low proportion of patients, i.e., $17 \%$ ( 63 out of 365 ) cotreated with cisplatin in cycle $1,14 \%$ (9 out of 63 ) of whom changed to carboplatin in later cycles. This introduces a bias in discriminating the covariate impact of platinum drug type. Although only paclitaxel impact was evaluated, the underlying hazard may partly be attributed to coadministered drugs, suggesting an overestimated impact of paclitaxel exposure in this clinically used drug combination treatment protocol in NSCLC.

In conclusion, we successfully developed a parametric cyclevarying hazard TTE model characterizing the time course of risk of first occurrence of clinically relevant paclitaxelassociated PN. Parametrically describing the baseline hazard of PN2+ enabled basing on mechanistic pharmacologic knowledge, e.g., time-dependent change in risk of PN2+ with change in paclitaxel exposure, to improve the characterization of observed PN2+ and enable better prediction of future incidences. FCM evaluation of covariate effects enabled better characterization of the impact of clinically relevant risk factors of $\mathrm{PN} 2+$, revealing older, male, and current smokers with high paclitaxel $\mathrm{AUC}_{\text {cycle }}$ as the highest-risk subpopulation and offering opportunity for prophylactic intervention or closer monitoring of symptoms for timely treatment. Modelbased comparisons suggested that reduction in risk of PN2+ was attainable through dose reduction rather than dose fractionation. The model enables prediction and comparison of individual patient's risks of $\mathrm{PN} 2+$ for different clinically relevant paclitaxel dosing schedules, facilitating dose selection to spare patients the disabling $\mathrm{PN} 2+$ and improve paclitaxel combination therapy.

\section{Acknowledgments}

The authors thank the Central European Society of Anticancer Drug Research for providing the study data and the High-performance Computing Services of Zentraleinrichtung für Datenverarbeitung (ZEDAT) at Freie Universitaet Berlin (https://www.zedat.fu-berlin. $\mathrm{de} / \mathrm{HPC} / \mathrm{EN} / \mathrm{Home}$ ) for the computational time.

\section{Authorship Contributions}

Participated in research design: Ojara, Henrich, Huisinga, Hartung, Joerger, Kloft.

Performed data analysis: Ojara.

Wrote or contributed to writing of the manuscript: Ojara, Henrich, Frances, Huisinga, Hartung, Joerger, Kloft.

\section{References}

Abraham JE, Guo Q, Dorling L, Tyrer J, Ingle S, Hardy R, Vallier AL, Hiller L, Burns

$\mathrm{R}$, Jones L, et al. (2014) Replication of genetic polymorphisms reported to be associated with taxane-related sensory neuropathy in patients with early breast cancer treated with paclitaxel. Clin Cancer Res 20:2466-2475.

Belani CP, Ramalingam S, Perry MC, LaRocca RV, Rinaldi D, Gable PS, and Tester WJ (2008) Randomized, phase III study of weekly paclitaxel in combination with carboplatin versus standard every-3-weeks administration of carboplatin and paclitaxel for patients with previously untreated advanced non-small-cell lung cancer. J Clin Oncol 26:468-473.

Boyette-Davis JA, Cata JP, Driver LC, Novy DM, Bruel BM, Mooring DL, Wendelschafer-Crabb G, Kennedy WR, and Dougherty PM (2013) Persistent chemoneuropathy in patients receiving the plant alkaloids paclitaxel and vincristine. Cancer Chemother Pharmacol 71:619-626.

de Graan AJ, Elens L, Sprowl JA, Sparreboom A, Friberg LE, van der Holt B, de Raaf PJ, de Bruijn P, Engels FK, Eskens FA, et al. (2013) CYP3A4*22 genotype and systemic exposure affect paclitaxel-induced neurotoxicity. Clin Cancer Res 19: 3316-3324.

Fountzilas G, Dafni U, Dimopoulos MA, Koutras A, Skarlos D, Papakostas P, Gogas H, Bafaloukos D, Kalogera-Fountzila A, Samantas E, et al. (2009) A randomized phase III study comparing three anthracycline-free taxane-based regimens, as first line chemotherapy, in metastatic breast cancer: a Hellenic Cooperative Oncology Group study. Breast Cancer Res Treat 115:87-99.

Gastonguay MR(2011) Full covariate models as an alternative to methods relying on statistical significance for inferences about covariate effects: a review of methodology and 42 case studies, in Population Approach Group Europe (PAGE); 2011 Jun 7-10; Athens, Greece. PAGE 20: 2229 [www.page-meeting.org/default.asp? abstract $=2229$ ]

Gornstein EL and Schwarz TL (2017) Neurotoxic mechanisms of paclitaxel are local to the distal axon and independent of transport defects. Exp Neurol 288:153-166. Green MC, Buzdar AU, Smith T, Ibrahim NK, Valero V, Rosales MF, Cristofanilli M, Booser DJ, Pusztai L, Rivera E, et al. (2005) Weekly paclitaxel improves pathologic complete remission in operable breast cancer when compared with paclitaxel once every 3 weeks. J Clin Oncol 23:5983-5992.

Henrich A, Joerger M, Kraff S, Jaehde U, Huisinga W, Kloft C, and Parra-Guillen ZP (2017) Semimechanistic bone marrow exhaustion pharmacokinetic/pharmacodynamic model for chemotherapy-Induced cumulative neutropenia. J Pharmacol Exp Ther 362:347-358.

Hershman DL, Lacchetti C, Dworkin RH, Lavoie Smith EM, Bleeker J, Cavaletti G, Chauhan C, Gavin P, Lavino A, Lustberg MB, et al.; American Society of Clinical Oncology (2014) Prevention and management of chemotherapy-induced peripheral neuropathy in survivors of adult cancers: American Society of Clinical Oncology clinical practice guideline. J Clin Oncol 32:1941-1967.

Holford N (2013) A time to event tutorial for pharmacometricians. CPT Pharmacometrics Syst Pharmacol 2:e43.

Joerger M, Kraff S, Huitema ADR, Feiss G, Moritz B, Schellens JH, Beijnen JH and Jaehde U (2012) Evaluation of a pharmacology-driven dosing algorithm of 3-weekly paclitaxel using therapeutic drug monitoring: a pharmacokineticpharmacodynamic simulation study. Clin Pharmacokinet 51:607-617.

Joerger M, von Pawel J, Kraff S, Fischer JR, Eberhardt W, Gauler TC, Mueller L, Reinmuth N, Reck M, Kimmich M, et al. (2016) Open-label, randomized study of individualized, pharmacokinetically (PK)-guided dosing of paclitaxel combined with carboplatin or cisplatin in patients with advanced non-small-cell lung cancer (NSCLC). Ann Oncol 27:1895-1902.

Johansson ÅM and Karlsson MO (2013) Multiple imputation of missing covariates in NONMEM and evaluation of the method's sensitivity to $\eta$-shrinkage. AAPS J 15: 1035-1042.

Kanbayashi Y, Hosokawa T, Kitawaki J, and Taguchi T (2013) Statistical identification of predictors for paclitaxel-induced peripheral neuropathy in patients with breast or gynaecological cancer. Anticancer Res 33:1153-1156.

Kawakami K, Tunoda T, Takiguchi T, Shibata K, Ohtani T, Kizu J, Nishio M, Horai T, Hama T, and Taguchi K (2012) Factors exacerbating peripheral neuropathy induced by paclitaxel plus carboplatin in non-small cell lung cancer. Oncol Res 20: 179-185.

Keizer RJ, van Benten M, Beijnen JH, Schellens JH, and Huitema AD (2011) Piraña and PCluster: a modeling environment and cluster infrastructure for NONMEM. Comput Methods Programs Biomed 101:72-79.

Kraff S, Nieuweboer AJ, Mathijssen RH, Baty F, de Graan AJ, van Schaik RH, Jaehde U, and Joerger M (2015) Pharmacokinetically based dosing of weekly paclitaxel to reduce drug-related neurotoxicity based on a single sample strategy. Cancer Chemother Pharmacol 75:975-983.

Lindbom L, Pihlgren P, and Jonsson EN (2005) PsN-Toolkit--a collection of computer intensive statistical methods for non-linear mixed effect modeling using NONMEM [published correction appears in Comput Methods Programs Biomed (2005) 80: 277]. Comput Methods Programs Biomed 79:241-257.

Lu D, Gillespie WR, Girish S, Agarwal P, Li C, Hirata J, Chu YW, Kagedal M, Leon L, Maiya V, et al. (2017) Time-to-event analysis of polatuzumab vedotin-induced peripheral neuropathy to assist in the comparison of clinical dosing regimens. $C P T$ Pharmacometrics Syst Pharmacol 6:401-408.

Marchetti P, Urien S, Cappellini GA, Ronzino G, and Ficorella C (2002) Weekly administration of paclitaxel: theoretical and clinical basis. Crit Rev Oncol Hematol 44 (Suppl):S3-S13.

Mehrotra S, Sharma MR, Gray E, Wu K, Barry WT, Hudis C, Winer EP, Lyss AP, Toppmeyer DL, Moreno-Aspitia A, et al. (2017) Kinetic-Pharmacodynamic model of chemotherapy-induced peripheral neuropathy in patients with metastatic breast cancer treated with paclitaxel, nab-paclitaxel, or ixabepilone: CALGB 40502 (Alliance). AAPS J 19:1411-1423.

Mielke S, Sparreboom A, Steinberg SM, Gelderblom H, Unger C, Behringer D, and Mross K (2005) Association of Paclitaxel pharmacokinetics with the development of peripheral neuropathy in patients with advanced cancer. Clin Cancer Res 11:4843-4850.

Otake A, Yoshino K, Ueda Y, Sawada K, Mabuchi S, Kimura T, Kobayashi E, Isobe A, Egawa-Takata T, Matsuzaki S, et al. (2015) Usefulness of duloxetine 
for Paclitaxel-induced peripheral neuropathy treatment in gynecological cancer patients. Anticancer Res 35:359-363.

Park SB, Kwok JB, Asher R, Lee CK, Beale P, Selle F, and Friedlander M (2017) Clinical and genetic predictors of paclitaxel neurotoxicity based on patient- versus clinician-reported incidence and severity of neurotoxicity in the ICON7 trial. Ann Oncol 28:2733-2740.

Plan EL, Ma G, Någård M, Jensen J, and Karlsson MO (2011) Transient lower esophageal sphincter relaxation pharmacokinetic-pharmacodynamic modeling: count model and repeated time-to-event model. J Pharmacol Exp Ther 339:878-885.

Rinker B, Fink BF, Barry NG, Fife JA, Milan ME, Stoker AR, and Nelson PT (2011) The effect of cigarette smoking on functional recovery following peripheral nerve ischemia/reperfusion injury. Microsurgery 31:59-65.

Rosen S, Ham B, and Mogil JS (2017) Sex differences in neuroimmunity and pain $J$ Neurosci Res 95:500-508.

Schuette W, Blankenburg T, Guschall W, Dittrich I, Schroeder M, Schweisfurth H, Chemaissani A, Schumann C, Dickgreber N, Appel T, et al. (2006) Multicenter randomized trial for stage IIIB/IV non-small-cell lung cancer using every-3-week versus weekly paclitaxel/carboplatin. Clin Lung Cancer 7:338-343.

Scripture CD, Figg WD, and Sparreboom A (2006) Peripheral neuropathy induced by paclitaxel: recent insights and future perspectives. Curr Neuropharmacol 4 $165-172$.

Seidman AD, Berry D, Cirrincione C, Harris L, Muss H, Marcom PK, Gipson G, Burstein H, Lake D, Shapiro CL, et al. (2008) Randomized phase III trial of weekly compared with every-3-weeks paclitaxel for metastatic breast cancer, with trastuzumab for all HER-2 overexpressors and random assignment to trastuzumab or not in HER-2 nonoverexpressors: final results of Cancer and Leukemia Group B protocol 9840. J Clin Oncol 26:1642-1649.

Sparano JA, Wang M, Martino S, Jones V, Perez EA, Saphner T, Wolff AC, Sledge GW Jr, Wood WC, and Davidson NE (2008) Weekly paclitaxel in the adjuvant treatment of breast cancer. N Engl J Med 358:1663-1671.

Stubblefield MD, Burstein HJ, Burton AW, Custodio CM, Deng GE, Ho M, Junck L, Morris GS, Paice JA, Tummala S, et al. (2009) NCCN task force report: management of neuropathy in cancer. J Natl Compr Canc Netw 7 (Suppl 5):S1-S26, quiz S27-S28.
Svensson EM, Svensson RJ, Te Brake LHM, Boeree MJ, Heinrich N, Konsten S, Churchyard G, Dawson R, Diacon AH, Kibiki GS, et al. (2018) The potential for treatment shortening with higher rifampicin doses: relating drug exposure to treatment response in patients with pulmonary tuberculosis. Clin Infect Dis 67 34-41.

Tanabe Y, Shimizu C, Hamada A, Hashimoto K, Ikeda K, Nishizawa D, Hasegawa J, Shimomura A, Ozaki Y, Tamura N, et al. (2017) Paclitaxel-induced sensory peripheral neuropathy is associated with an ABCB1 single nucleotide polymorphism and older age in Japanese. Cancer Chemother Pharmacol 79: 1179-1186.

Tarning J, Thana P, Phyo AP, Lwin KM, Hanpithakpong W, Ashley EA, Day NP, Nosten F, and White NJ (2014) Population pharmacokinetics and antimalarial pharmacodynamics of piperaquine in patients with plasmodium vivax malaria in Thailand. CPT Pharmacometrics Syst Pharmacol 3:e132.

Upton RN and Mould DR (2014) Basic concepts in population modeling, simulation, and model-based drug development: part 3-introduction to pharmacodynamic modeling methods. CPT Pharmacometrics Syst Pharmacol 3:e88.

Verdú E, Ceballos D, Vilches JJ, and Navarro X (2000) Influence of aging on peripheral nerve function and regeneration. J Peripher Nerv Syst 5:191-208.

Zhang J, Zhou F, Qi H, Ni H, Hu Q, Zhou C, Li Y, Baburina I, Courtney J, and Salamone SJ (2019) Randomized study of individualized pharmacokineticallyguided dosing of paclitaxel compared with body-surface area dosing in Chinese patients with advanced non-small cell lung cancer. $\mathrm{Br} J$ Clin Pharmacol 85: $2292-2301$.

Zhang S, Sun M, Yuan Y, Wang M, She Y, Zhou L, Li C, Chen C, and Zhang S (2016) Correlation between paclitaxel Tc $>0.05$ and its therapeutic efficacy and severe toxicities in ovarian cancer patients. Cancer Transl Med 2:131-136.

Address correspondence to: Charlotte Kloft, Department of Clinical Pharmacy and Biochemistry, Institute of Pharmacy, Freie Universitaet Berlin., Kelchstr. 31, 12169 Berlin, Germany. E-mail: charlotte.kloft@fuberlin.de 